\title{
NEW DETERMINATION OF THE POLAR MOTION FROM 1890 TO 1969
}

\author{
E. P. FEDOROV, A. A. RORSUN, S. P. MAJOR, \\ N. T. PANCHENKO, V. K. TARADY, and Ya. A. YAT SKIV \\ Ukrainian Academy of Sciences, Kiev, U.S.S.R.
}

\begin{abstract}
To obtain the coordinates of the Earth's pole almost all series of systematic latitude observations that continued for more than two years have been utilized. They are listed in Table I which comprises 92 series of observation at 72 observatories.

Computation was made by the following stages. As initial data we used normal values of latitude $\varphi_{1}, \varphi_{2}, \ldots \ldots \varphi_{n}$, i.e. the means of instantaneous latitudes over successive intervals of time. These values were smoothed using Whittaker's numerical method which is capable of giving the most probable curve of latitude variation. The smoothed values $\varphi^{\prime}$ satisfy the following condition
\end{abstract}

where

$$
\lambda^{2} H+G=\min .
$$

$$
H=\sum_{i=1}^{n-3}\left(\Delta^{3} \varphi_{i}\right)^{2}, \quad G=\sum_{i=1}^{n} h_{i}^{2}\left(\varphi_{i}-\varphi_{i}^{\prime}\right)^{2},
$$

$h_{i}$ is a measure of precision, $\lambda^{2}$ an arbitrary number by means of which the degree of smoothing is set, and $\Delta^{3}$ designates the third difference of $\varphi^{\prime}$. Whittaker's method was applied in different modifications according to whether or not the normal values of $\varphi_{i}^{\prime}$ had an equal weight and were given at equidistant moments of time.

For the origin of the system of coordinates we adopted the mean pole of the epoch of observation. Because of this the data given in Table II represent only the periodic part of the polar motion in the region of frequency from 0.77 to 2 cycles per year. In this connection the sequence of $\varphi^{\prime}$ was subjected to filtration in order to eliminate variation of the mean latitude.

Coordinates of the pole were computed in two approximations. First, it was assumed that all the series are of the same accuracy and so they were taken with an equal weight.

The polar coordinates obtained on this assumption are denoted by $x_{1}, y_{1}$ and shown in the second and third columns of Table II. The divergences of the smoothed values $\varphi_{i}^{\prime}$ from the latitudes computed with $x_{1}, y_{1}$ were denoted by $z_{\kappa i}$ where the index $\kappa$ designates the number of a series. Then for the second approximation each series of observation was taken with the weight inversely proportional to the mean value of $z_{\kappa i}^{2}$ for this series. The polar coordinates obtained in the second approximation are denoted by $x_{2}, y_{2}$ and given in the last two columns of Table II.

The full paper with the tables will be published by the Ukrainian Academy of Sciences as a separate book. 


\section{DISCUSSION}

R. O. Vicente: Besides the importance of local effects of the stations as emphasized by Fedorov, I agree with Fricke's comment that the errors in star positions are very important and therefore we should employ an up-to-date catalogue of the star positions for all the reductions.

E.P. Fedorov: Even the best meridian catalogues are not capable of giving the precision required for reduction of latitude observations, these observations themselves were used to improve declinations of observed stars which is a usual procedure. It is hoped that residual errors could be balanced out owing to the large number of series of observation.

P. Melchior: (1) Asks how the declination errors have been taken into account. (2) Says that the smoothing process using third order differences is a very good one. It depends upon the numerical value given to $\lambda$ parameter.

E. P. Fedorov: The same as to Prof. Vicente's question.

$W$. Fricke: I wonder whether Professor Fedorov thinks that the observations for determining the polar motion from 1890 to 1969 would be reduced to a common system.

E. P. Fedorov: The same as the answer to Prof. Vicente's question.

$H$. Jeffreys: At what intervals of time are the values of $\phi ;$ taken in forming the differences $\Delta^{3} \phi$ ?

E. P. Fedorov: If unequal intervals were dealt with divided differences were used. 